\title{
The prevalence of arterial and venous abnormalities in asymptomatic patients undergoing total knee arthroplasty
}

\author{
Halis Atıl Atilla (D), Mutlu Akdoğan (D) \\ Department of Orthopaedics and Traumatology, University of Health Sciences Dışkapı Ylldırım Beyazt Training and Research Hospital, Ankara, Turkey
}

\begin{abstract}
Objectives: This study aims to investigate the prevalence of venous and arterial diseases in asymptomatic patients undergoing total knee arthroplasty (TKA) and to evaluate possible surgery-related vascular complications.

Patients and methods: The data of a total of 96 extremities of 48 vascular asymptomatic patients $(9$ males, 39 females; mean age $65.7 \pm 51.8$ years; range, 50 to 85 years) who underwent arterial and venous bilateral Doppler ultrasound examination between October 2017 and February 2018 were retrospectively analyzed. Stenosis of the arterial and venous system thrombosis and venous insufficiency were recorded.

Results: Although no significant vascular pathologies such as deep vein thrombosis or arterial occlusion were detected, minor vascular pathologies such as minimal atherosclerosis (29.2\%) and venous reflux (25\%) were found in patients scheduled for TKA.

Conclusion: In most patients, TKA operations can be performed without a vascular comorbidity; however, even asymptomatic patients may carry vascular risks. These silent patients can be identified by simple non-invasive tests. Patients under risk are supposed to be consulted with vascular surgery before the operation and can be operated in the hospital setting where a vascular surgery unit is available. Recognizing the silent group with color Doppler ultrasound can be helpful and feasible in patients undergoing TKA to reduce complication rate and to take precautions.
\end{abstract}

Keywords: Arthroplasty, knee, pathology, peripheral, vascular.

Gonarthrosis is a degenerative non-inflammatory disease of the knee joint with cartilage damage, new bone formation, and deformity over the surface of the joint. Total knee arthroplasty (TKA) is the treatment of choice with the indication of gonarthrosis in elderly population. The majority of patients are elderly and, due to the natural course of the disease, individuals become sedentary. Therefore, comorbidities of cardiovascular system are expected in this patient group. On the other hand, TKA is one of the major orthopedic procedures and carries the risk of complications of arterial and venous systems. The rate of lower extremity venous thromboembolism (VTE) has been reported as 2.1 to $4.3 \%$ in patients undergoing TKA..$^{[1,2]}$ The rate of arterial complications has been shown to be 0.03 to $0.17 \%{ }^{[3,4]}$ Although the arterial risk rate seems to be low, the amputation risk is very high in case of arterial complications (up to 25 to $43 \%$ ). ${ }^{[5,6]}$

There is no consensus on a routine preoperative evaluation test to predict the risk of a possible vascular system complications in patients undergoing TKA. In the present study, we aimed to investigate the prevalence of venous and arterial diseases in asymptomatic patients undergoing TKA and to evaluate possible surgeryrelated vascular complications.

\section{PATIENTS AND METHODS}

The data of a total of 96 extremities of 48 asymptomatic patients ( 9 males, 39 females; mean age $65.7 \pm 51.8$ years; range, 50 to 85 years) who underwent arterial and venous bilateral color Doppler

Received: February 26, 2019 Accepted: March 31, 2019 Published online: July 25, 2019

Correspondence: Halis Atıl Atilla, MD. SBÜ Dışkapı Ylldırım Beyazıt Eğitim ve Araştırma Hastanesi Ortopedi ve Travmatoloji Kliniği, 06110 Dışkapı, Ankara, Turkey. e-mail: dratilatilla@hotmail.com 
ultrasonography (DUS) examination between October 2017 and February 2018 were retrospectively analyzed. Exclusion criteria were as follows: revision knee arthroplasties, being under the age of 50 and above 85 , having joint surgery from the same extremity within the past year, and previous diagnosis of peripheral arterial and venous system disease in patients with intermittent claudication, resting pain, and ulcus cruris at their extremities. Those who had percutaneous transluminal angioplasty or coronary artery bypass grafting or those with any malignancies were also excluded from the study. A written informed consent was obtained from each patient. The study protocol was approved by the institutional Ethics Committee (Date: 02.04.2018-No. 48/19). The study was conducted in accordance with the principles of the Declaration of Helsinki.

Routine arterial and venous DUS findings which were obtained in the preoperative period in TKA patients were examined. Stenosis of the arterial system and venous system thrombosis and venous insufficiency data were recorded. The relationship between age, sex, comorbidities, and arterial and venous pathologies was analyzed.

\section{Statistical analysis}

Statistical analysis was performed using the IBM SPSS for Windows version 20.0 software (IBM Corp., Armonk, NY, USA). Descriptive data were expressed in mean \pm standard deviation (SD) or number and frequency. For the quantitative data showing normal distribution, the independent sample test was used, while for the quantitative data showing non-normal distribution, the MannWhitney $U$ test was used. The chi-square test was used to compare qualitative data. A $p$ value of $<0.05$ was considered statistically significant with $95 \%$ confidence interval (CI).

\section{RESULTS}

In this study, 96 extremities of 48 patients were included. Baseline demographic characteristics of patients are shown in Table 1.

None of the patients had arterial occlusion requiring medical or surgical treatment. Minimal atherosclerotic plaques were found in 14 patients (29.2\%) and 28 extremities (29.1\%). All of these patients had bilateral atherosclerotic plaques. None of the patients had unilateral atherosclerosis. Distribution of arterial problems according to age are shown in Table 2.
Deep venous thrombosis (DVT) was not found in any patient. The only pathology involving the veins was venous reflux pathology in 12 patients (48\%) and 10 extremities (10.41\%). Distribution of venous problems according to age are also shown in Table 2.

Both arterial and venous pathologies were observed in eight patients. Arterial problems (i.e., atherosclerosis) were observed in all six extremities of male patients with venous problems. Of the female patients, four had arterial problems, while three had only venous problems.

The relationship between age and arterial and/or venous pathology was not found to be significant (for arterial pathologies $\mathrm{p}=0.096$ and for venous pathologies $\mathrm{p}=0.605)$. Similarly, the relationship between sex and arterial and venous pathology was not significant $(\mathrm{p}=0.708$ and $\mathrm{p}=0.671$, respectively). However, there was a significant correlation between age of male patients and venous pathology. In particular, there was an increase in the frequency of venous reflux in males with increasing age $(\mathrm{p}=0.048)$. This result was unable to be observed for arterial pathologies $(\mathrm{p}=0.167)$ (Table 2).

Fourteen patients (29.2\%) were free from comorbidities. Of 34 patients (70.8\%) with comorbidities, diabetes mellitus (DM) was present in $52.9 \%(n=18)$. Similarly, $52.9 \%(n=18)$ of the patients with comorbidities had hyperlipidemia. In $41.2 \%(n=14)$ of the patients with comorbidities, there were chronic diseases other than DM and hypercholesterolemia. In addition, many of the patients (70.8\%) who were scheduled for TKA had comorbidities which might cause vascular system pathologies. However, there was no statistically significant relationship between comorbidities and venous or arterial pathologies $(\mathrm{p}=1)$ (Table 3).

\section{DISCUSSION}

Vascular complications after TKA are very rare in the general population (0.05\%)..$^{[7,8]}$ However, in case of circulatory disorders due to vascular pathologies, there are several risks which may be fatal, ranging from

\begin{tabular}{lcccc}
\multicolumn{4}{l}{ Table 1. Baseline demographic characteristics of patients } \\
\hline \multicolumn{1}{c}{$\mathrm{n}$} & $\%$ & Mean & $p$ \\
\hline Age (year) & & & 65.73 & \\
Gender & & & & 0.946 \\
Male & 9 & 18.8 & 65.9 & \\
Female & 39 & 81.2 & 65.7 & \\
\hline
\end{tabular}




\begin{tabular}{|c|c|c|c|c|c|c|}
\hline & \multicolumn{3}{|c|}{ Present } & \multicolumn{3}{|c|}{ Absent } \\
\hline & $\mathrm{n}$ & $\%$ & Mean age & $\mathrm{n}$ & $\%$ & Mean age \\
\hline \multicolumn{7}{|c|}{ Atheroma plaque } \\
\hline Patient number & 14 & 29.2 & & 34 & 70.8 & \\
\hline \multicolumn{7}{|l|}{ Gender } \\
\hline Male & 2 & & 73.5 & 7 & 63.7 & \\
\hline Female & 12 & & 67.8 & 27 & 64.7 & \\
\hline \multicolumn{7}{|l|}{ Venous reflux } \\
\hline Patient number & 12 & 25 & & 36 & 75 & \\
\hline \multicolumn{7}{|l|}{ Gender } \\
\hline Male & $3 \mathrm{w}$ & & $74.3^{*}$ & 6 & & 61.7 \\
\hline Female & 9 & & $64.2^{*}$ & 30 & & 66.1 \\
\hline
\end{tabular}

\begin{tabular}{|c|c|c|c|c|c|c|c|}
\hline & \multicolumn{2}{|c|}{ Present } & \multicolumn{2}{|c|}{ Absent } & \multicolumn{2}{|c|}{ Total } & \multirow[b]{2}{*}{$p$} \\
\hline & $\mathrm{n}$ & $\%$ & $\mathrm{n}$ & $\%$ & $\mathrm{n}$ & $\%$ & \\
\hline \multicolumn{8}{|c|}{ Atheroma plaque } \\
\hline Absent & 10 & 20.8 & 24 & 50 & 34 & 70.8 & \multirow{3}{*}{0.954} \\
\hline Present & 4 & 8.4 & 10 & 20.8 & 14 & 29.2 & \\
\hline Total & 14 & 29.2 & 34 & 70.8 & 48 & 100 & \\
\hline \multicolumn{8}{|c|}{ Venous reflux } \\
\hline Absent & 11 & 22.9 & 25 & 52.1 & 36 & 75 & \multirow{3}{*}{0.714} \\
\hline Present & 3 & 6.3 & 9 & 18.7 & 12 & 25 & \\
\hline Total & 14 & 29.2 & 34 & 70.8 & 48 & 100 & \\
\hline
\end{tabular}

wound closure problems to acute ischemic stroke and pulmonary embolism. ${ }^{[9]}$ Most patients with vascular complications after TKA are those had previous peripheral vascular disease. Therefore, peripheral vascular evaluation is recommended in many centers before and after TKA. ${ }^{[8]}$

The three main factors associated with VTE include positive VTE history, varicose veins, and congestive heart failure after hip or knee arthroplasty. ${ }^{[10]}$ These three main reasons are the diseases which can give evidence with color DUS and are helpful to inform the surgeon in terms of the preoperative measures. Tsuji et al. ${ }^{[11]}$ in their study in which they attempted to establish a quantitative index of pre-TKA protective anticoagulant therapy, they highlighted the importance of preoperative vascular ultrasound and found that one of the three risk factors which increased DVT after TKA was the maximum soleus vein diameter on ultrasonographic examination. Although DVT was not detected in any case in our study, there was a statistically significant relationship between venous reflux and saphenous vein proximal diameter $(\mathrm{p}=0.038$ [R], 0.025[L]).

Park et al. ${ }^{[12]}$ reported a total of 692 patients with TKA who underwent preoperative DUS. In their study, vascular pathology was found in $4.6 \%$ of patients and arterial atherosclerotic changes in $3.6 \%$ of patients. The authors found DVT in only two of 1,000 extremities. In our study, the rate of venous pathology was $39.5 \%$ and atherosclerotic changes were seen in $31.2 \%$. The significant difference in these ratios may be due to the fact that even minimal atherosclerotic changes were reported, if seen on DUS in our study. Yet, the rate of atherosclerotic plaque encountered in the general population was similar to our study. Hirsch et al. ${ }^{[13]}$ found that $29 \%$ of the patients had peripheral arterial disease in a study performed by a first-line screening.

In another study, De Laurentis et al. ${ }^{[14]}$ reported the first study investigating the incidence of peripheral obstructive arterial disease in patients undergoing arthroplasty. They evaluated arterial disease by ankle brachial index and plethysmography and found a prevalence of $2 \%$. The rate of patients with postoperative arterial complications was reported to be $0.5 \%$ in this study. The main issue in this study was that all patients who experienced all complications were in this $2 \%$-patient group during screening. Complications can be predicted and reduced by determination of preoperative vascular risks of patients. After the 
anatomical and physiological screening of patients with color DUS, patients with a positive risk can be applied the ankle arm index and the patients at a higher risk can be identified.

Acute popliteal artery occlusion from arterial complications is rarely reported in the literature and has often been reported as case reports. ${ }^{[15,16]}$ In patients with atherosclerosis, the acute rupture of atheroma plaque is the main cause and has been reported as 0.03 to $0.17 \% .^{[16]}$ Preliminary diagnosis of risky patients is critical to identify the urgent need for intervention after or during TKA. Another benefit is that, in such centers where vascular surgeons do not work, this type of risky patients are not supposed to operated and referred to large centers. Although the complication risk is rare, the results can be very serious as amputation, embolism, and even death due to venous and arterial thrombus formation. Early arterial problems after TKA can be treated by open thrombectomy, bypass surgery, or endovascular methods. ${ }^{[17-19]}$

Chang et al. ${ }^{[20]}$ reported preoperative DVT incidence as $2.6 \%$ per patient and $1.6 \%$ per knee by computed tomography/indirect venography. Preoperative prevalence and postoperative incidence of major DVT were reported as $2.5 \%{ }^{[12]}$ In the study of Wakabayashi et al. ${ }^{[21]}$ including 322 TKA patients, 56 patients $(17.4 \%)$ had DVT in their preoperative DUS. They reported that the risk of DVT increases significantly in patients with rheumatoid arthritis (RA) and connective tissue diseases. ${ }^{[21]}$ In our study group, patients with various risks and symptoms such as RA connective tissue disease, previous surgery and previously known vascular disease were excluded from the study, and probably due to the relatively small number of patients in our study, none of our patients had preoperative DVT. Other causes which increase the risk of VTE after TKA were reported as female sex, age ( $\geq 80$ years), hypertension, existing malignancy, obesity (body mass index $\geq 30 \mathrm{~kg} / \mathrm{m}^{2}$ ), and black race. ${ }^{[11]}$ Venous pathologies were less common in women than in male patients (M/F 33.3/23.1\%). Hypertension and obesity were not in the exclusion criteria in the study, although patients with malignant diseases were not included in this study either. It is more reasonable to identify the risk of peripheral vascular disease in female patients and those with obesity and hypertension.

Even if total TKA is performed unilaterally, bilateral evaluation of vascular pathology is important, as the coagulation cascade in the contralateral extremity can be activated as a systemic condition after surgery. ${ }^{[22]}$ Regardless of the operative side, the patients who had venous and arterial color DUS before TKA on both sides were included in the current study.

In this study, we investigated the prevalence of peripheral vascular disease of asymptomatic patients by color DUS. As another method, the risk of peripheral vascular disease can be detected radiographically. ${ }^{[23]}$ Vascular calcifications may be a predictor of ischemic complications in patients with TKA. Patients with intimal type calcification should be treated carefully and close follow-up of the postoperative vascular condition is recommended. On the other hand, a non-calcified atheroma plaque has a more unstable structure and the calcified plaque is less likely to have a rupture than the calcified plaque. ${ }^{[23]}$ Therefore, it would be appropriate to evaluate the vessels with color DUS which can detect even soft plaques.

The main limitations of the present study include its retrospective single-center and design and relatively small sample size. Multicenter prospective randomized studies with larger patient series would help to demonstrate the risk of pre-TKA vascular pathology in asymptomatic patients.

In conclusion, preliminary detection of possible vascular pathologies in TKA may help to minimize risks. Atherosclerosis and venous reflux rates increase with age in male patients; therefore, the risk of vascular pathology should be carefully investigated in elderly men who are scheduled for TKA. Color DUS can be performed safely in routine examinations prior to TKA to provide information about the anatomical and physiological conditions of both the arteries and veins. This method is cost-effective, harmless, non-invasive, and available even in small-scale hospitals, depending on the availability of radiologists. The vast majority of patients scheduled for TKA can be operated with a low risk of peripheral vascular system complications. Operation of the patients at a higher risk group in centers where cardiovascular surgery units are available can provide early detection, prevention, and treatment of peripheral vascular complications through close follow-up and early examination of the patients in the early postoperative period.

\section{Declaration of conflicting interests}

The authors declared no conflicts of interest with respect to the authorship and/or publication of this article.

\section{Funding}

The authors received no financial support for the research and/or authorship of this article. 


\section{REFERENCES}

1. Hamilton WG, Reeves JD, Fricka KB, Goyal N, Engh GA, Parks NL. Mechanical thromboembolic prophylaxis with risk stratification in total knee arthroplasty. J Arthroplasty 2015;30:43-5.

2. Falck-Ytter Y, Francis CW, Johanson NA, Curley C, Dahl $\mathrm{OE}$, Schulman S, et al. Prevention of VTE in orthopedic surgery patients: Antithrombotic Therapy and Prevention of Thrombosis, 9th ed: American College of Chest Physicians Evidence-Based Clinical Practice Guidelines. Chest 2012;141:278-325.

3. Calligaro KD, DeLaurentis DA, Booth RE, Rothman RH, Savarese RP, Dougherty MJ. Acute arterial thrombosis associated with total knee arthroplasty. J Vasc Surg 1994;20:927-30.

4. Cho MR, Kim KT, Choi WK. Arterial occlusion after total knee arthroplasty despite minimal invasive technique in aneurysm at popliteal artery: Case report. Medicine (Baltimore) 2018;97:e12719.

5. He R, Yang L. Acute arterial occlusion in the midpiece of femoral artery following total knee arthroplasty: Report of one case. Chin J Traumatol 2016;19:116-8.

6. Kumar SN, Chapman JA, Rawlins I. Vascular injuries in total knee arthroplasty. A review of the problem with special reference to the possible effects of the tourniquet. J Arthroplasty 1998;13:211-6.

7. Calligaro KD, Dougherty MJ, Ryan S, Booth RE. Acute arterial complications associated with total hip and knee arthroplasty. J Vasc Surg 2003;38:1170-7.

8. Abu Dakka M, Badri H, Al-Khaffaf H. Total knee arthroplasty in patients with peripheral vascular disease. Surgeon 2009;7:362-5.

9. Menendez ME, Greber EM, Schumacher CS, Lowry Barnes C. Predictors of Acute Ischemic Stroke After Total Knee Arthroplasty. J Surg Orthop Adv 2017;26:148-153.

10. Zhang J, Chen Z, Zheng J, Breusch SJ, Tian J. Risk factors for venous thromboembolism after total hip and total knee arthroplasty: a meta-analysis. Arch Orthop Trauma Surg 2015;135:759-72.

11. Tsuji S, Kobayashi A, Tomita T, Hamada M, Sugamoto K, Yoshikawa H. Quantitative index for deciding whether to administer preventive anticoagulant therapy in osteoarthritis patients undergoing total knee arthroplasty. J Orthop Sci 2014;19:77-84.

12. Park IH, Lee SC, Park IS, Nam CH, Ahn HS, Park HY, et al. Asymptomatic peripheral vascular disease in total knee arthroplasty: preoperative prevalence and risk factors. J Orthop Traumatol 2015;16:23-6.

13. Hirsch AT, Criqui MH, Treat-Jacobson D, Regensteiner JG, Creager MA, Olin JW, et al. Peripheral arterial disease detection, awareness, and treatment in primary care. JAMA 2001;286:1317-24.

14. DeLaurentis DA, Levitsky KA, Booth RE, Rothman RH, Calligaro KD, Raviola CA, et al. Arterial and ischemic aspects of total knee arthroplasty. Am J Surg 1992;164:23740.

15. Park BJ, Cho HM, An KY, Lee HJ. Acute Arterial Occlusion Following Primary Total Knee Arthroplasty. Knee Surg Relat Res 2018;30:84-8.

16. Cho MR, Kim KT, Choi WK. Arterial occlusion after total knee arthroplasty despite minimal invasive technique in aneurysm at popliteal artery: Case report. Medicine (Baltimore) 2018;97:e12719.

17. Reynolds A, Sandstrom A, Jha PK. Totally Endovascular Management of Popliteal Artery Occlusion and Pseudoaneurysm Formation after Total Knee Replacement. Ann Vasc Surg 2017;38:316.

18. Tsujimoto R, Matsumoto T, Takayama K, Kawakami Y, Kamimura M, Matsushita T, et al. Acute Popliteal Artery Occlusion after Revision Total Knee Arthroplasty. Case Rep Orthop 2015;2015:672164.

19. Chikkanna JK, Sampath D, Reddy V, Motkuru V. Popliteal Artery Thrombosis after Total Knee Replacement: An Unusual Complication. J Clin Diagn Res 2015;9:1-2.

20. Chang MJ, Song MK, Kyung MG, Shin JH, Chang CB, Kang SB. Incidence of deep vein thrombosis before and after total knee arthroplasty without pharmacologic prophylaxis: a 128-row multidetector CT indirect venography study. BMC Musculoskelet Disord 2018;19:274.

21. Wakabayashi H, Hasegawa M, Niimi R, Yamaguchi T, Naito Y, Sudo A. The risk factor of preoperative deep vein thrombosis in patients undergoing total knee arthroplasty. J Orthop Sci 2017;22:698-702.

22. Walls RJ, O'Malley J, O'Flanagan SJ, Kenny PJ, Leahy AL, Keogh P. Total knee replacement under tourniquet control: A prospective study of the peripheral arterial vasculature using colour-assisted duplex ultrasonography. Surgeon 2015;13:303-7.

23. Woelfle-Roos JV, Dautel L, Wernerus D, Woelfle KD, Reichel H. Vascular Calcifications on the Preoperative Radiograph: Predictor of Ischemic Complications in Total Knee Arthroplasty? J Arthroplasty 2016;31:1078-82. 